\title{
Langasite-based Microbalance for Determination of the Non-stoichiometry in Praseodymium-Cerium Oxide Thin Films at Elevated Temperatures

\author{
\begin{tabular}{r|r|r} 
Sebas & Non- \\
Clausthal University of Technology, & Stoichiometry Stolle \\
Sebasti & $\begin{array}{l}\text { Fritze } \\
\text { search and Physical Technologies, } \\
\text { Germany } \\
\text { sthal.de } \\
\text { Praseodymiu } \\
\text { m-Cerium }\end{array}$
\end{tabular}
}

\section{Abstract:}

The oxygen partial pressure dependent non-stoichiometry of praseodymium-cerium oxide thin films $\left(\operatorname{Pr}_{0.1} \mathrm{Ce}_{0.9} \mathrm{O}_{2-\delta}, \mathrm{PCO}\right)$ is investigated at temperatures between 700 and $900{ }^{\circ} \mathrm{C}$. The non-stoichiometry of PCO is determined gravimetrically using a high-temperature microbalance consisting of piezoelectric langasite $\left(\mathrm{La}_{3} \mathrm{Ga}_{5} \mathrm{SiO}_{14}\right)$ resonators. Shifts of the resonance frequency are correlated with mass changes caused by oxygen incorporation/release using the Sauerbrey equation and converted into the oxygen non-stoichiometry of PCO.

Chemical and mechanical properties of the PCO films might influence the resonance frequency. Single-side deposition of PCO onto the resonators results in calculated non-stoichiometry values which exceed literature data for bulk PCO. A potential explanation is mechanical bending of the resonators due to thermo-chemical expansion. Resonators coated on both sides with PCO show smaller frequency shifts corresponding to a non-stoichiometry of up to $\delta=0.07$, which are close to literature data.

Key words: Microbalance, high-temperature sensor, piezoelectric resonator, thin films, oxygen nonstoichiometry, oxygen ion pump

\section{Motivation}

In general, metal oxide thin films are used in electronic devices, e.g. MEMS $\left(\mathrm{SiO}_{2}, \mathrm{Al}_{2} \mathrm{O}_{3}\right)$, micro-batteries $\left(\mathrm{LiMnO}_{2}\right)$ or sensors $\left(\mathrm{SnO}_{2}\right.$ $\mathrm{TiO}_{2}$, etc.). Metal oxides, particularly those containing transition metals or rare earth ions with multiple oxidation states, can achieve considerable oxygen non-stoichiometry which impacts the materials properties [1]. Therefore, the investigation of the non-stoichiometry in metal oxide thin films is of major interest to widen its applicability range.

Praseodymium-cerium oxide (PCO) is a promising material for oxygen storage in three-way catalysts, for cathodes of solid oxide fuel cells [2] or for mass dependent gas sensing applications [3]. Especially for the latter two applications, $\mathrm{PCO}$ is operated in the form of thin films at temperatures above $500{ }^{\circ} \mathrm{C}$.

So far, numerous investigations focus on bulk samples but the results cannot be transferred to thin films or porous samples due to their higher surface to volume ratio. The enthalpy of reduction is lower for thin films. As a result, the nonstoichiometry of thin films exceeds bulk values [4]. Nevertheless, the correlation of oxygen non-stoichiometry and defect mechanisms in thin films are not fully understood yet, especially in the medium oxygen partial pressure range (approximately $10^{-6}$ bar to $10^{-15}$ bar). Consequently, further investigations are required.

Conventional thermogravimetric analysis of PCO films is not feasible due to the low sample mass. A quartz crystal microbalance enables to detect mass changes in the order of nanogram, but the phase transformation from $\alpha$ - to $\beta$ quartz at $573{ }^{\circ} \mathrm{C}$ prevents high-temperature measurements. In addition, material-related losses in $\alpha$-quartz limit the operation for sensor application to about $450{ }^{\circ} \mathrm{C}$ [5]. Therefore, Y-cut langasite $\left(\mathrm{La}_{3} \mathrm{Ga}_{5} \mathrm{SiO}_{14}, \mathrm{LGS}\right)$ single crystals are chosen as a high-temperature stable piezoelectric transducer. LGS is a quartz-isomorph which shows no phase transformation up to its melting point at $1470{ }^{\circ} \mathrm{C}$. Most important, LGS can be piezoelectrically excited to bulk acoustic shear waves up to temperatures close to its melting point $[6,7,8]$.

\section{Defect Chemistry of PCO}

The defect chemistry of $\mathrm{Pr}_{\mathrm{x}} \mathrm{Ce}_{1-\mathrm{x}} \mathrm{O}_{2-\delta}$ can be described by a model presented e.g. in [2]. The non-stoichiometry depends on the equilibrium 
between the oxygen in the lattice and the surrounding gas atmosphere as well as the oxidation pairs $\mathrm{Pr}^{3+} / \mathrm{Pr}^{4+}$ and $\mathrm{Ce}^{3+} / \mathrm{Ce}^{4+}$. The incorporation/release of oxygen follows eq. (1) given here using the Kröger-Vink notation.

$$
2 \mathrm{Pr}_{\mathrm{Ce}}^{\mathrm{x}}+\mathrm{O}_{\mathrm{O}}^{\mathrm{x}} \rightleftharpoons 2 \mathrm{Pr}_{\mathrm{Ce}}^{\prime}+\mathrm{V}_{\mathrm{O}}+1 / 2 \mathrm{O}_{2(\mathrm{~g})}
$$

The $p_{\mathrm{O} 2}$ dependent oxygen vacancy concentration $\left[\mathrm{V}_{\mathrm{O}}^{*}\right]$ and, therefore, the non-stoichiometry can be divided in three different regimes with respect to the dominating defects. At high $p_{\mathrm{O} 2}$, the release of oxygen is compensated by reduction of $\mathrm{Pr}^{4+}$ to $\mathrm{Pr}^{3+}$ resulting in eq. (2)

$\left[\mathrm{V}_{\mathrm{O}}\right] \sim \mathrm{p}_{\mathrm{O} 2}^{-1 / 6}$

At medium $p_{\mathrm{O} 2}$, when all $\mathrm{Pr}$ ions are trivalent, the oxygen vacancy concentration is fixed to fulfill the neutrality condition. At low $p_{\mathrm{O} 2}, \mathrm{Ce}^{4+}$ starts to reduce to $\mathrm{Ce}^{3+}$ and even more oxygen is released. For this work, the first and second regime are of particular interest. The nonstoichiometry $\delta$ is defined in eq. (3)

$$
\delta=\left[\mathrm{V}_{0}\right] /\left[\mathrm{Pr}_{0.1} \mathrm{Ce}_{0.1} \mathrm{O}_{2}\right]
$$

\section{Langasite-based Microbalance}

Applying an $\mathrm{AC}$ voltage close to the mechanical resonance frequency of a piezoelectric $\mathrm{Y}$-cut LGS single crystal excites thickness shear bulk acoustic waves [5]. Thereby, platinum/rhodium key hole shaped films deposited via pulse laser deposition serve as electrodes. LGS resonators, layer sequences and dimensions are shown Fig. 1. The deposition of mass $\Delta \mathrm{m}$ in form of a film or a change in stoichiometry of a thin film already deposited onto the resonator shift the resonance frequency $(\Delta f)[9]$. Eq. (4) is valid for mass changes up to $2 \%$ of the resonators mass if no other effects impact the resonance frequency.

$\Delta \mathrm{f} / \mathrm{f}=-\Delta \mathrm{m} / \mathrm{m} \rightarrow \Delta \mathrm{m}=-\mathrm{A} \Delta \mathrm{f} / \mathrm{s}$

In eq. (4), $A$ is the effective resonator area and $S(S=-A \partial f / \partial m)$ the mass sensitivity [5].

The frequency at the conductance maxima (series resonance frequency $f\left(G_{\text {max }}\right)=f_{\text {res }}$ ) is tracked since it is independent of stray capacities [5]. In addition, the conductance spectra in the vicinity of the resonance frequency can be approximated by a Lorentzian function [8], which is beneficial for data evaluation. Since the resonance frequency is strongly temperature dependent $\left(\partial \mathrm{f}_{\text {res }} / \partial \mathrm{T} \sim 280 \mathrm{~Hz} \mathrm{~K}^{-1}\right.$ at $600{ }^{\circ} \mathrm{C}$ for a $5 \mathrm{MHz}$ resonator), temperature fluctuations of even $0.01 \mathrm{~K}$ could overwhelm the signal of interest. Therefore, internal temperature compensation according to [5] is applied. It uses the resonance frequency of different vibrational modes (e.g. $1^{\text {st }}$ and $3^{\text {rd }}$ ) to calculate a temperature compensated resonance frequency $f_{\mathrm{IC}}$, see eq. (5).

$f_{I C}(m, \ldots)=f^{[1]}(T, m, \ldots)-\frac{c^{[1]}}{c^{[3]}} f^{[3]}(T, m, \ldots)$

In eq. (5), $c^{[1]}, c^{[3]}$ are the temperature coefficients of the fundamental mode and the $3^{\text {rd }}$ overtone, respectively. As a consequence, the temperature compensated mass sensitivity $S_{I C}$ of the resonator needs to be recalculated, see eq. (6).

$S_{I C}=S^{[1]}\left(1-3 \frac{C^{[1]}}{C^{[3]}}\right)$

The mass resolution $R$ depends on the mass sensitivity $S$ and the accuracy of the resonance frequency determination. Taking the experimental conditions into account (thin-film diameter: $5 \mathrm{~mm}, 5 \mathrm{MHz}$ resonator at $700{ }^{\circ} \mathrm{C}$ : $\Delta f^{[1]}= \pm 3 \mathrm{~Hz}$ [5] and $\Delta f_{\mathrm{IC}}= \pm 9 \mathrm{~Hz}$ ) the mass resolution is about $180 \mathrm{ng}$. Stable operation of LGS is ensured in hydrogen containing atmospheres down to oxygen partial pressure of $10^{-20}$ bar and $10^{-13}$ bar at $600^{\circ} \mathrm{C}$ and $800^{\circ} \mathrm{C}$, respectively [5].

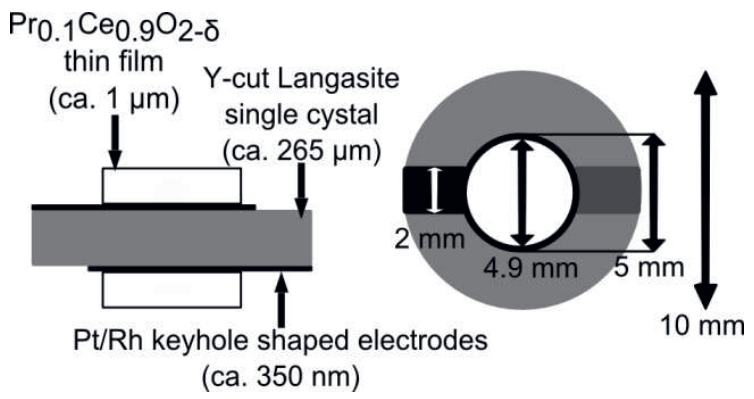

Fig. 1. Layers and dimension of a resonator; LGS single crystal, electrodes and PCO thin film.

\section{Experimental}

Polished Y-cut LGS single crystals are purchased from SICCAS (China). Platinum electrodes and PCO films are deposited by pulsed laser deposition using a KrF-Excimer laser (248 nm, COMPex, Lambda Physics, Germany). The PCO targets are prepared by stoichiometric mixtures of $\mathrm{Pr}_{6} \mathrm{O}_{11} \quad(99.9 \%$, Matek $\mathrm{GmbH}$, Germany) and $\mathrm{Ce}(\mathrm{IV}) \mathrm{O}_{2} \quad(99.99 \%$, Chempur $\mathrm{GmbH}$, Germany) powders which are uniaxially pressed with $55 \mathrm{MPa}$ and sintered at $1350{ }^{\circ} \mathrm{C}$. Film thicknesses are measured by profilometry using a stylus profiler XP2 (Ambios Technologies, USA).

Crystal structure of the PCO thin films is confirmed by X-Ray diffractometry (XRD) using a Siemens D5005 (Siemens AG, Germany) with a copper cathode. The set up matches the BraggBretano geometry. A Nickel filter decreases the 
intensity of the $\mathrm{Cu}_{\mathrm{k}-\beta}$ radiation. The resolution is $0.01^{\circ}$.

An oxygen ion pump based on yttrium stabilized zirconia enables the precise adjustment of $p_{\mathrm{O} 2}$ in the range between 0.2 and $10^{-24}$ bar. Oxygen ion transport is driven by a pump current. $A$ sensor in the vicinity of the sample enables PID control of the $p_{\mathrm{O} 2}$ with an accuracy of $\Delta \log \left(p_{\mathrm{O} 2} /\right.$ bar $)<0.02$ [11]. The set up of the system is shown in fig 2 . The main advantage of the oxygen ion pump is the precise adjustment of the oxygen partial pressure in the medium $p_{\mathrm{O} 2}$ range, which is interesting for sensors in regard of the application limit. Commonly, high $p_{\mathrm{O} 2}$ are adjusted by mass flow control of oxygen and e.g. nitrogen or argon and low $p_{\mathrm{O} 2}$ by buffer gas mixtures, e.g. $\mathrm{H}_{2} / \mathrm{H}_{2} \mathrm{O}$, but medium $p_{\mathrm{O} 2}$ are not easily accessible. The oxygen ion pump is connected to the gas reservoir ( $\mathrm{Ar}$ gon $(99.996 \%), 5 \% \mathrm{H}_{2} / \operatorname{Ar}(99.99 \%)$ and oxygen $(99.5 \%)$ for $p_{\mathrm{O} 2}>10^{-3}$ bar). Mass flow controller (MKS Instruments, Germany) guarantees a constant gas flow of $20 \mathrm{~cm}^{3} \mathrm{~min}^{-1}$.

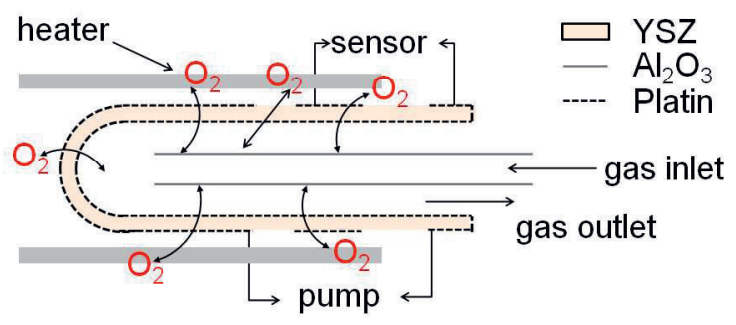

Fig. 2 Set-up of the oxygen ion pump (acc. to [11]).

The $p_{\mathrm{O} 2}$ dependent non-stoichiometry is measured at 700 and $900{ }^{\circ} \mathrm{C}$ in a gas tight tube furnace. A thermocouple (type $B$ ) and an oxygen sensor are installed in the vicinity of the resonator to control temperature and $p_{\mathrm{O} 2}$. The piezoelectric resonators are mounted in an alumina sample holder. A network analyzer (E5100 A, Agilent, USA) acquires the impedance spectra of the resonators. For the measurement of the resonance frequency, a three point calibration (open, shot and resistance of $47.3 \Omega$ ) is applied. Computer software allows an automatic tracking of the resonance frequency. All spectra contain 400 data points. The $p_{\mathrm{O} 2}$ is varied stepwise between $10^{-1}$ bar and $10^{-8}$ bar. Each $p_{\mathrm{O} 2}$ is held for $10-20 \mathrm{~h}$ to accomplish a steady state condition for the resonance frequency. The resonance frequency, oxygen partial pressure and temperature are measured every 10-15 s.
A scheme of the data recording and evaluation is given in fig. 3 .

\section{Results and Discussion}

Tab. 1 summarizes the key sample properties, i.e. film mass, film thickness and temperature compensated mass sensitivities of the resonators. In this context, the term "sample" refers to the resonator with the thin film. Resonators 1 and 2 are coated on side, only. Resonator 3 is coated on both sides (see tab. 1). Temperature coefficients are obtained by a linear fit in a temperature range of $\pm 5 \mathrm{~K}$ of the measurement temperature.

Tab. 1: Film mass and thickness of PCO and temperature compensated mass sensitivity of the resonators.

\begin{tabular}{|c|c|c|c|}
\hline Sample & $\begin{array}{c}\text { Film } \\
\text { mass } \\
{[\mu \mathrm{g}]}\end{array}$ & $\begin{array}{c}\text { Total film } \\
\text { thickness } \\
{[\mathrm{nm}]}\end{array}$ & $S_{\mathrm{IC}}$ \\
{$\left[\mathrm{cm}^{2} \mathrm{~Hz} \mathrm{\mu g}^{-1}\right]$} \\
\hline 1 & 63.5 & $642 \pm 45$ & $-18.1\left(700^{\circ} \mathrm{C}\right)$ \\
\hline 2 & 67.8 & $563 \pm 47$ & $0.7\left(900^{\circ} \mathrm{C}\right)$ \\
\hline 3 & 117.1 & $\begin{array}{c}1995 \pm \\
110\end{array}$ & $\begin{array}{c}-27.1\left(700^{\circ} \mathrm{C}\right) \\
4.3\left(900^{\circ} \mathrm{C}\right)\end{array}$ \\
\hline
\end{tabular}

Calculated densities $\left(\rho=\Delta m / A_{R} \cdot d\right)$ with $A_{R}$ is the thin film radius applying Sauerbrey model and $d$ is the film thickness) of the thin film are $6.9 \mathrm{~g} \mathrm{~cm}^{-3}$ in average and therefore close to the bulk value $\left(7.31 \mathrm{~g} \mathrm{~cm}^{-3}\right.$ [2]). Profilometry results in a surface roughness of about $100 \mathrm{~nm}$ which indicates high surface areas.

XRD shows a diffraction peak at about $28.6^{\circ}$, which can be assigned to the (111) reflex of fluorite crystal structure of PCO [12]. The peak occurs after annealing the samples at $700{ }^{\circ} \mathrm{C}$, only.

It must be noted that the temperature compensated resonance frequency $f_{\mathrm{IC}}$ and mass sensitivity $S_{I C}$ (see tab. 1) can show negative values, depending on the ratio of the temperature coefficients of the corresponding vibrational modes. Nevertheless, the mass change is reflected correctly. Fig. 4 shows the resonance frequency $\left(1^{\text {st }}\right.$ and $3^{\text {rd }}$ vibrational mode) of a both-sided coated resonator as function of temperature in air. The resonance frequencies follow a parabolic dependence (red lines). Differences between the heating and cooling are insignificant. 


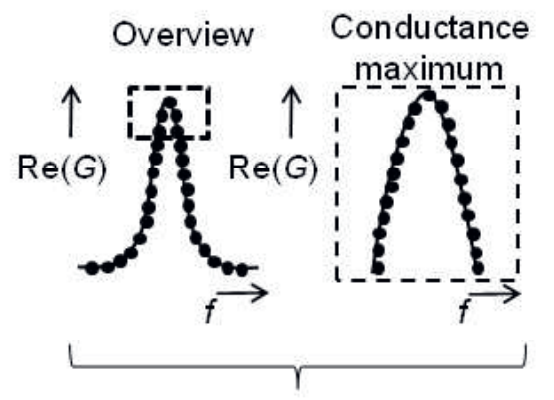

Tracking of the resonance frequency

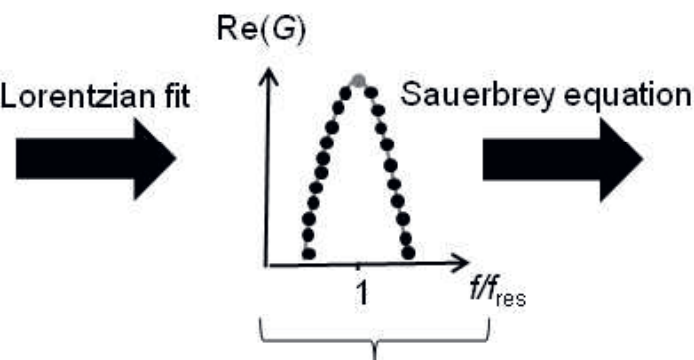

Fit with Lorentzian function to determine the resonance frequency and calculation of $f_{\mathrm{IC}}$

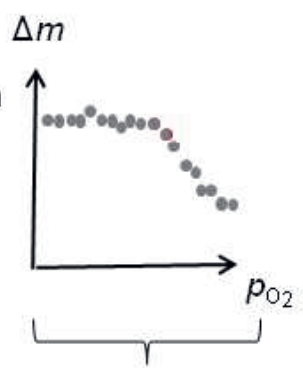

Correlation and plot of $\Delta m v s p_{\mathrm{O}_{2}}$

Fig. 3 Scheme of the data recording and evaluation

Therefore it can be concluded that temperature effects on the PCO thin film are reversible in air.

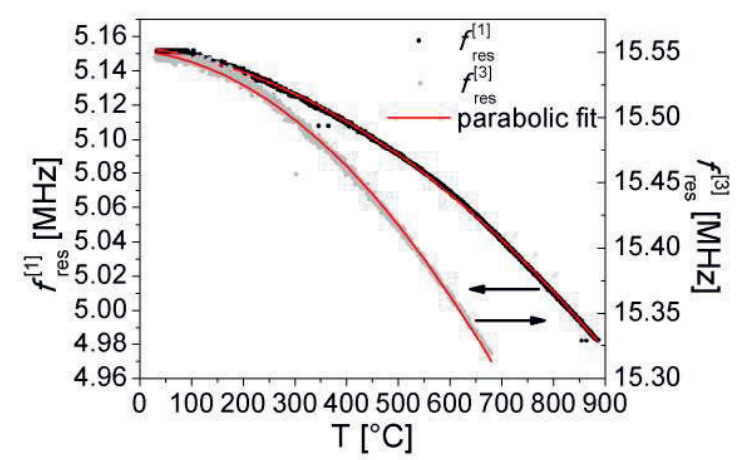

Fig. 4 Temperature dependence of the $1^{\text {st }}$ and $3^{\text {rd }}$ vibrational mode of a both-sided coated resonator

Changes of the oxygen partial pressure result in a sudden change of the resonance frequency, but it takes $10-20 \mathrm{~h}$ until steady-state conditions are achieved. Furthermore, the fluctuation of the data causes an uncertainty in determination of the evaluation of steady-state conditions.

Samples 1 and 2 show higher fluctuations of the resonance frequency, especially for the calculated internal compensated resonance frequency. The internal compensated mass sensitivities (see tab.1) are low (e.g. the mass sensitivity of a $5 \mathrm{MHz}$ resonator is about $32.1 \mathrm{~cm}^{2} \mathrm{~Hz} \mathrm{ug}^{-1}$ [5]) due to the ratio of $c^{[1]}$ and $c^{[3]}$. As a result, applying eq. (6) results in higher errors. Calculated mass (and nonstoichiometry) changes exceed literature data. For sample 2, the calculated mass loss during decreasing the $p_{\mathrm{O} 2}$ to $10^{-8}$ bar exceeds the film mass itself. Therefore, applying Sauerbrey equation is obviously a wrong interpretation and a more comprehensive examination for influences on the resonance frequency is required. A potential explanation might be the thermo- chemical expansion of $\mathrm{PCO}$, which results in mechanical stress at the interface of the electrode and the PCO thin film [1]. A Bending of the planar resonator could, potentially, cause a resonance frequency shift much larger than for pure mass changes.

Resonators coated on both sides show results which are at $700{ }^{\circ} \mathrm{C}$ in good agreement with literature data. In contrast to single-side coated resonators, bending of the resonators and, therefore, additional frequency shift is minimized.

The measurement at $900{ }^{\circ} \mathrm{C}$ results again in too high values for the mass losses due to the low internal compensated mass sensitivity. Evaluation of the $1^{\text {st }}$ mode and $f_{\mathrm{IC}}$ results in mass changes up to $10 \mu \mathrm{g}$ and $90 \mu \mathrm{g}$, respectively. This indicates that the impact of thin films stress/bending of the resonator is different for the $1^{\text {st }}$ and $3^{\text {rd }}$ mode.

Fig. 5 shows the oxygen partial pressure dependent frequency shifts and corresponding mass changes for sample 2 at $700^{\circ} \mathrm{C}$. The resonance frequency at $p_{\mathrm{O} 2}=10^{-1}$ bar and $700{ }^{\circ} \mathrm{C}$ is taken as the reference for further calculation. The non-stoichiometry and mass loss (according to eqs. (3) and (4)) increases initially and reaches a plateau at about $10^{-5}$ bar as predicted by the defect model [2]. The literature data presented in fig. 5 refer to a pure oxygen atmosphere at room temperature. The calculation of our data is done using literature data at high $p_{\mathrm{O} 2}$ as reference. 

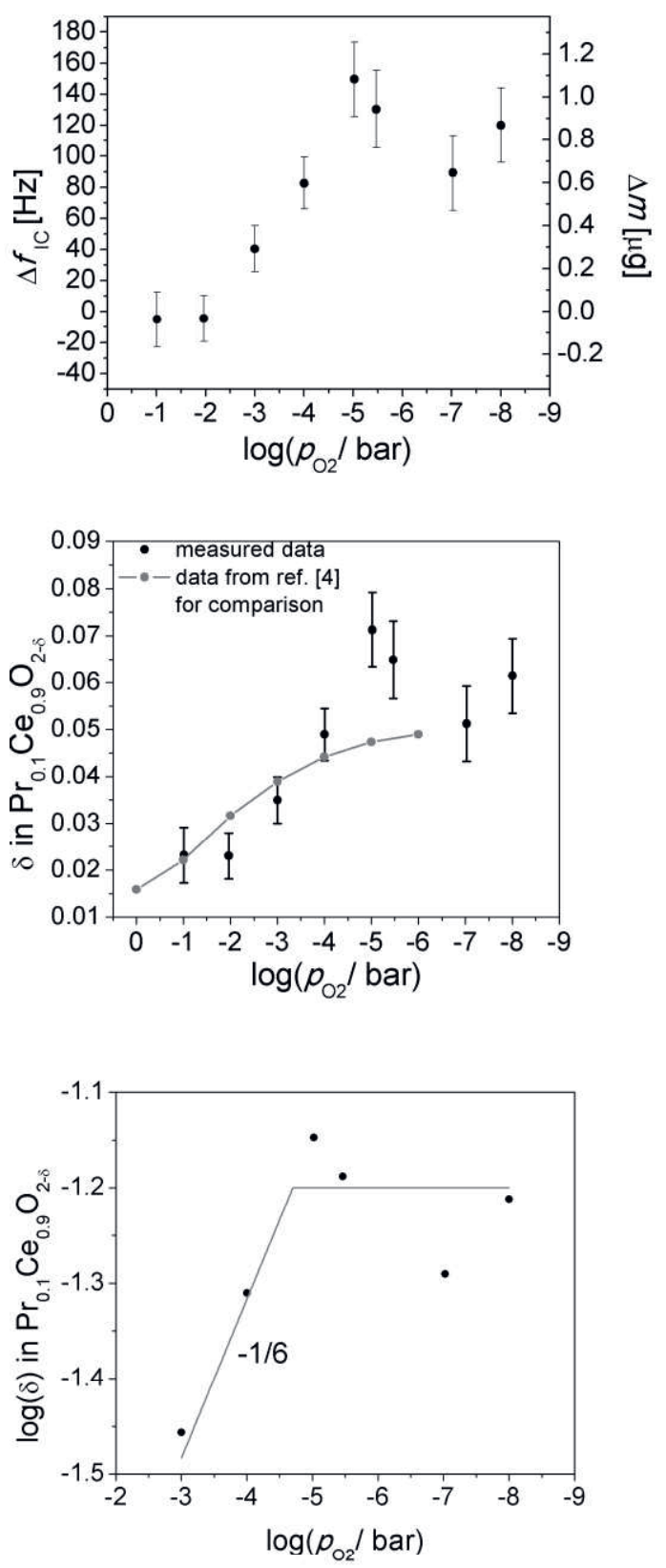

Fig.5. Shift of the temperature compensated resonance frequency and calculated mass changes (top) and non-stoichiometry (middle) of resonator 3 at $700{ }^{\circ} \mathrm{C}$. Error bars for the oxygen partial pressure are smaller than data points. The logarithm of the nonstoichiometry in dependence of the oxygen partial pressure is given for comparison with the defect model (bottom). Data points at $10^{-1}$ bar and $10^{-2}$ bar are excluded because of errors bars include $\delta=0$. The grey line reflects the defect model and does not represent a fit of the data.

Compared to the fundamental mode and $3^{\text {rd }}$ overtone, the temperature compensated resonance frequency shows more fluctuations.

Reasons for the deviation of calculated nonstoichiometries and literature values are in ac- cordance with the ability of thin films to show higher non-stoichiometries compared to bulk samples. The behavior results from a decreased enthalpy of reduction of praseodymium [4] and decreased activation energy for the incorporation/release of oxygen [13] in such films. Another indication is the mass loss in porous gadolinium doped ceria (bulk samples), which exceeds values for dense samples [14]. Recently, a coupling of strain, stress and nonstoichiometry in thin film PCO is presented in [1]. Non-stoichiometry results in a shift of the defect formation energies, which appears to decrease with tensile stress (up to about $750{ }^{\circ} \mathrm{C}$ ) and increase with compressive stress (above $750^{\circ} \mathrm{C}$ ) [1]. The thermo-chemical expansion coefficient decreases with increasing temperature and the elastic modulus is greater than in bulk samples. In addition, stress relaxation and slow kinetics are observed [1]. Comprehensive stress could be a reason why measurements at $900{ }^{\circ} \mathrm{C}$ exceed reasonable values even for both side coated resonators. In addition, irreversible stresses are observed in this work and in [1] causing difficulties in data evaluation.

\section{Summary and Conclusion}

The non-stoichiometry in praseodymium-cerium oxide thin films $\left(\mathrm{Pr}_{0.1} \mathrm{Ce}_{0.9} \mathrm{O}_{2-\delta}, \mathrm{PCO}\right)$ is investigated by a high-temperature stable microbalance (Y-cut langasite, $\mathrm{La}_{3} \mathrm{Ga}_{5} \mathrm{SiO}_{14}$ ).

Mass changes of the thin film as function of the oxygen partial pressure are measured by monitoring the resonance frequency and applying Sauerbrey equation. Since the resonance frequency is strongly temperature dependent, a temperature compensation based on measuring the fundamental mode and third overtone according to [5] is applied. It is assumed that mass changes are result of release/ incorporation of oxygen.

PCO thin films are prepared by stoichiometric mixing of $\mathrm{Pr}_{6} \mathrm{O}_{11}$ and $\mathrm{Ce}(\mathrm{IV}) \mathrm{O}_{2}$ powders, which are uniaxially pressed and sintered at $1350{ }^{\circ} \mathrm{C}$. The received pellets are used for pulsed laser deposition process. After a heat treatment at $700{ }^{\circ} \mathrm{C}$ in air, XRD indicates fluorite crystal structure of the thin films. The resonance frequency shows insignificant changes during heat treatment in air, indicating that thermal effects are reversible.

Calculated non-stoichiometries for thin films exceed literature values for bulk samples, which is a common phenomenon and a result of a decreased enthalpy of reduction of praseodymium and activation energy for the incorporation/release of oxygen. 
Mechanical stress caused by the thin films might effect the resonance frequency. Resonators coated with PCO on both sides and measured at $700{ }^{\circ} \mathrm{C}$ show good agreement with the above mentioned literature data.

\section{Acknowledgement}

The authors thank the Energie Forschungszentrum (Energy Research Centre) for financial support.

\section{References}

[1] J. Sheth, D. Chen, J. J. Kim, W. J. Bowman, P. A. Crozier, H. L. Tuller, S. T. Misture, S.

Zdzieszynski, B. W. Sheldon, S. R. Bishop, Coupling of strain, stress, and oxygen nonstoichiometry in thin film $\mathrm{Pr}_{0.1} \mathrm{Ce}_{0.9} \mathrm{O}_{2-\delta}$, Nanoscale 8, 16499-16510 (2016); doi: 10.1039/c6nr04083g

[2] S.R. Bishop, T.S. Stefanik, H. L. Tuller, Electrical conductivity and defect equilibria of $\mathrm{Pr}_{0.1} \mathrm{Ce}_{0.9} \mathrm{O}_{2-\delta}$, Physical Chemistry and Chemical Physics 13, 10165-10173 (2011); doi: 10.1039/COCP02920C

[3] H. Seh, H. L. Tuller, H. Fritze, Thin film Praseodymium-Cerium Oxide Langasite based microbalance gas sensor, Journal of Electroceramics 13, 755-778 (2004); doi: 10.1007/s10832-0045191-y

[4] D. Chen, S. R. Bishop, H. L. Tuller, Nonstoichiometry in oxide thin films operating under anodic conditions: A chemical capacitance study of the Praseodym-Cerium oxide system, Chemistry of Materials 26, 6622-6627 (2014); doi: $10.1021 / \mathrm{cm} 503440 \mathrm{v}$

[5] H. Fritze, High-temperature bulk acoustic wave sensors, Measurement Science and Technology 22, 01200-012030 (2011); doi: 10.1088/0957$0233 / 22 / 1 / 012002$

[6] D. Richter, H. Fritze, T. Schneider, P. Hauptmann, N. Bauersfeld, K.-D. Kramer, K. Wiesner, M. Fleischer, G. Karle, A. Schubert, Integrated high temperature gas sensor system based on bulk acoustic wave resonators, Sensors and Actuators B 118, 466-471 (2006) doi: 10.1016/j.snb.2006.04.041

[7] M. Schultz, H. Fritze, Electromechanical properties of langasite resonators at elevated temperatures, Renewable Energy 33, 336-341 (2008); doi: 10.1016/jrenene.2007.05.016

[8] H. Fritze, H. L. Tuller, G. Borchardt, T Fukuda, High-Temperature Properties of Langasite, MRS Proceedings 604, 65-70 (1999); doi: 10.1557/PROC-604-65

[9] G. Sauerbrey, Verwendung von Schwingquarzen zur Wägung dünner Schichten und Mikrowägung, Zeitschrift für Physik 155, 206-222 (1959)

[10] A. Bund, G. Schwitzgebel, Signal-oscillations of a piezoelectric quartz crystal in liquids caused by compressional waves, Analytica Chimica Acta 364, 189-194 (1998); doi: 10.1016/S00032670(98)00201-3
[11] M. Schulz, J. Brillo, C. Stenzel and H. Fritze, Oxygen partial pressure control for microgravity experiments, Solid State Ionics 225, 332-336 (2012); doi: 10.1016/j.ssi.2012.04.008

[12] K. Ahn, D. S. Yoo, D. H. Prasad, W.-W. Lee, Y.C- Chung, J.H. Lee, Role of multivalent Pr in the formation and migration of oxygen vacancy in $\mathrm{Pr}$ doped Ceria: Experimental and First-principles investigations, Chemistry of Materials 24, 42614267 (2012); doi: 10.1021/cm3022424

[13] J.J. Kim, S. R. Bishop, N. J. Thompson, D. Chen, $H$. L. Tuller, Investigation of nonstoichiometry in oxide thin films by simultaneous in situ optical absorption and chemical cpacitance measurements: pr-doped Ceria, a case study, Chemistry of Materials 26, 1374-1379 (2014); doi: $10.1021 / \mathrm{cm} 403066 \mathrm{p}$

[14] S. R. Bishop, K. L. Duncan, E. D. Wachsman, Surface and bulk oxygen non-stoichiometry and bulk chemical expansion in gadolinium-doped cerium oxide, Acta Materialia 57, 3596-3605 (2009); doi: 10.1016/jactamat.2009.04.017

[15] B. W. Sheldon, V. B. Shenoy, Space charge induced surface stress: Implication in Ceria and other ionic solids, Physical Review Letters 106, 216104-216108 (2011); doi: 10.1103/PhysRevLett.106.216104 